\title{
Modeling Variability in Reaching Motions
}

\author{
Julian J. Faraway \& Jennifer Hu \\ Department of Statistics \\ University of Michigan
}

April 2001

This article was presented at the SAE Digital Human Modeling Conference, Arlington, VA, June 26-28, 2001.

\section{ABSTRACT}

Motion prediction models may give the average reach for an individual of specified characteristics. The actual reach will vary from this reach in a manner that may depend on both systematic and random factors. We describe a modeling approach that incorporates the variability within the reaches of a given subject and that between subjects. This information is useful to designers in investigating phenomena that may not occur during the average reach but may occur during variants such as collision with an obstacle or injury due to over-exertion.

\section{INTRODUCTION}

Repeated reaches by the same individual to the same location will show some variability. Reaches by different individuals will show even greater variability. Some of this increased variability will be due to observable characteristics of these individuals such as gender or age. We can adjust for this by considering individuals with the same external characteristics such as height, weight, age or gender or any other factor believed to make a systematic difference. Even then, these externally identical individuals will show even greater variation in their reaches to the same location than the repeated reaches of a single individual due to unmeasurable or unsuspected personal differences. This article shows how to model and understand these two types of variation in reaching motions.

There are three main reasons for modeling these variabilities. The first concerns the practical value of this knowledge. For example, a model that predicts the hand trajectory might be used to design a layout to avoid collisions. The predicted trajectory for a reach to a given location might avoid a collision. However, we know that in repeated reaches, the hand trajectory is likely to vary and if a collision occurs in some fraction of these repeated reaches, we may wish to change the layout. Furthermore, some injuries occur because a motion is performed in an unusual or unexpected way. With a model for the variability, we can predict the probabilities of motions that differ from the average. Thus it is sometimes not sufficient to simply predict the average way a 
given motion might be performed. We must also understand how repeated motions might vary about this average and how these motions might vary from subject to subject.

The second reason for pursuing this approach concerns model validity. A model might predict how an individual of specified characteristics might perform a given reach. We know that when the individual actually performs the reach, the observed and predicted reaches will not be identical. This does not mean that the model is invalid since some variation in reaching is to be expected. Nevertheless, it is important that a model tell us how much variation from the predicted motion is to be expected. Without this information, it will be difficult to tell whether the differences between observed and predicted motions can be explained by modeled variability or whether they represent shortcomings in the model itself.

Another model validity issue arises when the model is used for some new purpose. Models built using experimental subjects will be used to predict the motion of new subjects outside the laboratory. Even if these new subjects match the characteristics of the experimental subjects, we cannot expect that these new subjects will perform in an identical way. A model that ignores this between subject variability will claim greater precision than the facts will justify. Of course, it is possible that the model will not work well because conditions in the field differ from those in the laboratory that cause some systematic difference in the motion. However, it is necessary to distinguish the reasons for differences between the observed and predicted motions. Some differences will simply be because the subjects are different. We aim to measure and model this variation so that users of the model will know how much to expect.

The third reason for incorporating variability into the model concerns the statistical properties. Consider three scenarios: A: a single subject performs a given reach 100 times, B: 100 subjects perform the reach once, $\mathrm{C}$ : 10 subjects perform the reach 10 times each. In each case, there will be 100 reaches in the dataset but the amount of information available in each case will be different. The repeated reaches of a single individual will be more similar than the reaches of different individuals even if these individuals have the same external characteristics such as stature and weight. A will tell us only about variation within an individual while B tells us only about variation between individuals. $\mathrm{C}$ tells us something about both. The modeling approach should reflect this fact. Because it is often difficult to correctly allow for the grouping structure, it is commonly ignored. However, the computational hardware and software is now available to model this correctly and it is no longer necessary to cut corners.

We describe below a modeling approach that predicts reaching motions given information about the target of the reach and characteristics of the individual such as stature, age and gender. The model can output a predicted reach for given input conditions and it can express the likely variability in that reach. The amount of variability may depend on the input conditions such as the gender of the subject. The approach is used to model some data collected at the University of Michigan which we describe below. Although the analysis does provide some insight into variability in reaching motions, this article primarily aims to present the statistical methodology for studying this variability and not a comprehensive study of actual variability.

There has been some prior work on variability in motion. For example, [8] proposed a theory of motor-output variability which explains how the movement error is related to the movement amplitude, movement time, and the mass to be moved. [1] investigated if velocity profiles are invariant for grasp movements of different speed and whether kinematic variability is associated with movement speed and practice. [4] found that the variability time profiles can be expanded on 
a special system of basic functions corresponding to established movement parameters under the assumption that variability is defined solely by parameter variations.

\section{DATA}

In 1998, the Human Motion Simulation Laboratory (HUMOSIM) at the University of Michigan conducted a set of experiments concentrated on the motions of seated people performing right handed reaches to a spatially, well dispersed set of targets. The hand motion began from either in front of the person on a small table, or in the 2 o'clock position on a steering wheel, and proceeded to specified target locations, paused for a few seconds, and returned to the initial position. The reaches to the target and the return reaches were modeled separately. A total of about 8000 motions were performed by a group of 20 subjects.

The subjects were selected to provide a means to assess the effects of anthropometry (height in particular), gender, and age on the motions. The subjects ranged from very short to very tall and from 20 to 60 years of age. Three different seating environments were examined: a car seat with medium side bolsters, a bus/light truck seat, and an industrial seat with a narrow backrest. During the industrial seated reaches, motions were performed with and without a light hand load (which was set to load the shoulder at between $6 \%$ to $15 \%$ of extended arm shoulder strengths).

Two different motion capture systems were simultaneously used to estimate joint angle changes throughout the motions: an optical reflective marker system (Qualysis MacReflex) and an electromagnetic (Ascension Flock-of-Birds). A seven-link kinematic model was created with joint centers estimated from the captured motion data. Joint angles were computed by processing the motion data through a version of JACK $(\mathrm{tm})$ software configured to estimate 19 global and 11 local angles.

The first step of the data analysis is to extract the portions of the recorded motion where the subject was reaching. We computed the distance of the finger from its rest position and used this to determine the beginning and end of the reach. There is a pause when the target is reached, the duration of which is random and may be different at different times. We have no particular interest in this so we removed these periods also. We divide the motion into reaching and returning parts.

We emphasize that the methodology presented here could be applied to data collected in different ways for different linkages. We have provided some details of the data collection to allow the reader to follow the examples below but the data and the conclusions drawn from this data are not the focus of this article.

\section{FUNCTIONAL REGRESSION MODEL}

First we describe the basic functional regression model that does not include any special variance structure. More details on this approach may be found in [3] from which the following description is derived.

After extracting the data, for any selected angle, or other measure, and a specified motion, we have a sequence of observed values from the start to the end of the motion. These sequences are of different lengths because some targets are further away than others and people reach at different speeds. For example, consider an axis joining the initial and final location of the hand. We can compute the orthogonal distance of the hand from this axis during motion. We shall call this 
distance the radial deviation. We have chosen to focus on this measure in the analysis below, but the same methodology could be applied to other measures.

Plots of this distance for 20 subjects reaching with the right hand to a location somewhat to the left and front of the body and about the same height as the initial position of the hand are shown in Figure 1. We need to standardize the lengths of the curves if we are to compare different reaches. We define $t=0$ to be the start of the motion and $t=1$ to be the end of the motion.

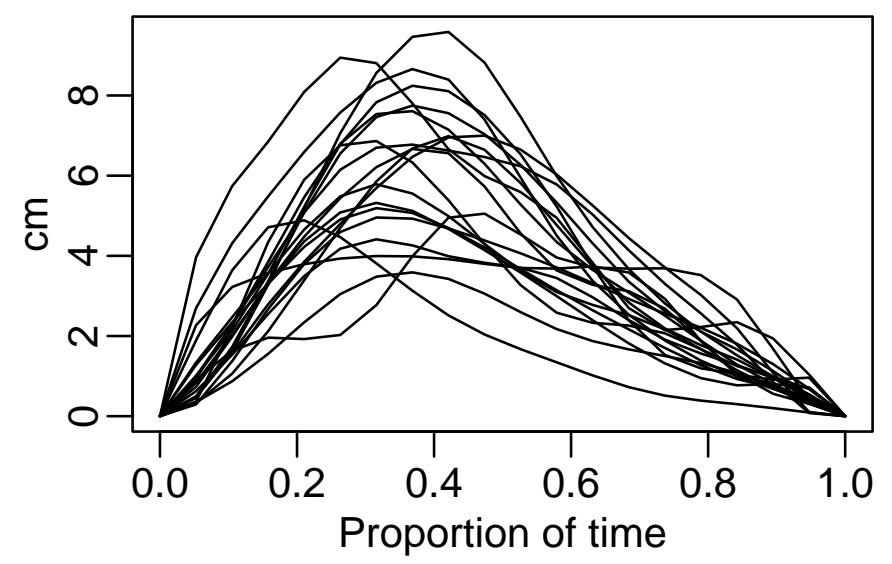

Figure 1: The radial deviation of the hand from a straight line path when reaching with the right hand to a location on the left for 20 subjects

We can describe each motion in terms of a set of functions. Some of these functions may describe how given angles change over time while others may describe how other quantities like the radial deviation change. There are many choices of angles and other quantities that could be used to describe the motion. We do not aim to say anything about which particular choice may be best, merely that such a set of functions does exist that can completely describe the motion. We aim to build a set of models that can predict each of these functions and thus predict the complete motion itself.

Suppose the rescaled functions for some chosen quantity are given by $\mathbf{y}(\mathbf{t})=\left(y_{1}(t), \ldots y_{n}(t)\right)$. These functions might be expected to depend on certain covariates such as the location of the target being reached, the age and anthropometry of the subject and other factors. For the $i^{t h}$ subject, we collect these predictors in a vector $x_{i}$. Typically, the first term in this vector is one. We then propose a functional linear model for the functions:

$$
y_{i}(t)=x_{i}^{T} \beta(t)+\varepsilon_{i}(t)
$$

Notice that this is comparable to a standard regression model but the response is now a function as is the error term $\varepsilon_{i}(t)$. The regression coefficients $\beta(t)$ are now a vector of functions. A general introduction to the area of functional data analysis may be found in [7]. The particular coefficient function for a given covariate will now represent the effect on the response of that covariate over the duration of the reach. We can now estimate $\beta(t)$ using the method of least squares applied across the whole time period. The estimator takes a familiar form:

$$
\hat{\beta}(t)=\left(X^{T} X\right)^{-1} X^{T} \mathbf{y}(t)
$$


where $X$ is the matrix whose rows are given by the $x_{i}$ 's.

Although this is conceptually illuminating, it is not practical since we cannot observe a $y_{i}(t)$ at all possible $t$. One approach is to approximate the functions on a grid of values. This was done in [2] with further developments in [9]. The major drawback is that a fine grid of values is necessary for accurate representation and so large matrices of values are required. These can be burdensome to manipulate and store.

For the current data, we have taken a basis function approach. We use standard cubic Bsplines. We represent the curves as linear combinations of these basis functions, $\psi_{j}(t)$. A curve $y_{i}(t)$ is represented as

$$
y_{i}(t)=\sum_{j=1}^{m} y_{i j} \psi_{j}(t)+\varepsilon_{i}(t)
$$

where the coefficients $y_{i j}$ are found by minimizing

$$
\int_{0}^{1}\left(y_{i}(t)-\sum_{j=1}^{m} y_{i j} \psi_{j}(t)\right)^{2} d t
$$

We observe $y(t)$ at some set of time points and so the coefficients $y_{i j}$ can be estimated using least squares. It is not uncommon for some parts of the curve to be missing due to data collection problems. This method can tolerate a certain amount of missing data. Furthermore, if measurement or processing systems are prone to producing outliers, a robust fitting method may be substituted for least squares. Given that human motion is usually quite smooth, it is not necessary to have a large number of basis functions. In this particular application, we found that eight basis functions were adequate. Note that eight grid points would not have been adequate for the approach mentioned above so this allows for a large reduction in the data needed for fitting these models and in the representation of the predictive models.

Using more than eight basis functions did not improve the fit significantly and, furthermore, restricting the number of coefficients has the advantage of smoothing out any small irregularities due to measurement error. We chose cubic B-splines for the basis because of their well-known stability for numerical calculations in contrast to polynomials. The cubic degree also allows for continuous first and second derivatives which is important if velocity and acceleration are needed.

Thus we can write the model in the approximate form

$$
Y_{n \times m} \psi_{m \times 1}(t)=X_{n \times p} B_{p \times m} \psi_{m \times 1}(t)+\varepsilon_{n \times 1}(t)
$$

or factoring out the $\psi(t)$, we can write it in the simpler form:

$$
Y_{n \times m}=X_{n \times p} B_{p \times m}+\varepsilon_{n \times m}
$$

which is now a multivariate multiple regression model where the coefficient matrix $B$ may be simply estimated using least squares as in

$$
\hat{B}=\left(X^{T} X\right)^{-1} X^{T} Y
$$

We may then use the standard methods of statistical inference using this modelling approach. Details of such methods may be found in texts such as [5]. For prediction and interpretation 
purposes, it is necessary to transform back from this basis function representation to the original form. For example, for a particular $\hat{\beta}(t)$ we would need to take a linear combination of cubic splines represented by the appropriate row of $\hat{B}$. Explicitly, we may recover the original coefficient functions by

$$
\hat{\beta}_{p \times 1}(t)=\hat{B}_{p \times m} \psi_{m \times 1}(t)
$$

and predict future responses given a predictor value $x_{0}$ by

$$
\hat{y}_{0}(t)=x_{01 \times p} \hat{B}_{p \times m} \psi_{m \times 1}(t)
$$

We estimate the variance of the prediction by

$$
\operatorname{vâ} r\left(\hat{y}_{0}(t)\right)=\psi^{T}(t) \hat{\Sigma} \psi(t) x_{0}^{T}\left(X^{T} X\right)^{-1} x_{0}
$$

where

$$
\hat{\Sigma}=\frac{\hat{\varepsilon}^{T} \hat{\varepsilon}}{n-p} \quad, \quad \hat{\varepsilon}=Y-X \hat{B}
$$

\section{Mixed Effects Functional Model}

The previous analysis would be fine provided that each reach were an independent motion. However, this is not the case since only 20 subjects were used in the study and reaches by the same individual will likely show some correlation. A model based on the approach above will be satisfactory for predicting motion but it cannot give an accurate view of the variation that will be present.

Consider one particular quantity such as the radial deviation. Let $y_{i j k}$ be the $k^{\text {th }} \mathrm{B}$-spline coefficient of the $i^{t h}$ subject performing the $j^{t h}$ reach. Let $x_{i j}$ be a vector containing the values of the predictors for the $j^{t h}$ reach of subject $i$. Some of these values may be associated with the subject such as stature and others may be associated with the reach such as the coordinates of the target. Yet other values might involve interactions between these two types of term. Typically, the first element of $x_{i j}$ will be one to allow for an intercept term. We start with the simplest model that contains all the pertinent elements:

$$
y_{i j k}=x_{i j}^{T} \beta_{k}+\gamma_{i}+\gamma_{i j}+\varepsilon_{i j k}
$$

This model differs from that described above due to the inclusion of so-called random effect terms $\gamma_{i}$ and $\gamma_{i j}$. We have

$$
\gamma_{i} \sim N\left(0, \sigma_{s}^{2}\right), \quad \gamma_{i j} \sim N\left(0, \sigma_{r}^{2}\right), \quad \varepsilon_{i j k} \sim N\left(0, \sigma^{2}\right)
$$

The $\gamma_{i}$ term represents the subject variation and is simply modeled as an additive term generated from a normal distribution with mean zero and variance $\sigma_{s}^{2}$. No loss of generality is implied by setting this mean to zero since any non-zero amount could be incorporated into the fixed effects term $x_{i j}^{T} \beta_{k}$. Clearly this is a simplistic view of how subject variation will enter and we show how to elaborate this later. The $\gamma_{i j}$ term represents the variation within reaches and is modeled as an additive term generated from a mean zero, variance $\sigma_{r}^{2}$ normal distribution. Again, we are modeling this reach variability as an additive perturbation. Finally we have the usual error term $\varepsilon_{i j k}$ which 
will incorporate the measurement error and other unexplained variation. We assume that all three error terms are independently generated. We show how to relax this assumption later.

Despite its simplicity, this model implicitly incorporates correlation between the B-spline coefficients. The correlation between the response (the B-spline coefficients) for observations on the same subject but different reaches is $\sigma_{s}^{2} /\left(\sigma_{s}^{2}+\sigma_{r}^{2}+\sigma^{2}\right)$ while the correlation between the coefficients of the same reach is $\left(\sigma_{s}^{2}+\sigma_{r}^{2}\right) /\left(\sigma_{s}^{2}+\sigma_{r}^{2}+\sigma^{2}\right)$. Thus the hierarchical nature of the random effects terms induces a correlation between the responses. This ensures that the reaches of the same subject will show a greater similarity than the reaches of different subjects.

As mentioned above, some elaboration of this model is desirable. For example, the predictors may affect the variability as well as the mean predicted motion. Such effects are included in the following model:

$$
y_{i j k}=x_{i j}^{T} \beta_{k}+z_{i} \gamma_{i k}+z_{i j} \gamma_{i j k}+\varepsilon_{i j k}
$$

Here $z_{i}$ is a vector of predictors associated with subject $i$. It might include values such as the subject's stature and gender. We may model the possibility of variability changing as a function of these values. The vector $z_{i j}$ contains values related to the reach $j$ and possibly interacting with the subject $i$. For example, it is possible that reaches to particular locations might be more variable than others and that the variation might depend on gender. $x$ and the $z$ 's may contain some predictors in common but they need not be identical. $\gamma_{i k}$ is now a vector of random effects where we allow for the possibility that the effects may also vary with the coefficient number $k$. We assume $\gamma_{i k} \sim N\left(0, \psi_{s}\right)$ where the covariance matrix $\psi_{s}$ must be estimated. This matrix may take a completely general positive definite form or we might impose some restriction (like diagonality) to reduce the number of parameters to be estimated. Similarly, $\gamma_{i j k} \sim N\left(0, \psi_{r}\right)$. We can also allow the distribution of $\varepsilon$ to be more general. For example, we might expect more variation in the middle of the motion. This could be modeled by allowing $\varepsilon_{i j k} \sim N\left(0, \sigma_{k}^{2}\right)$.

The models above can be fit using restricted maximum likelihood methods (REML). We have used the software of [6]. The datasets considered here are fairly large and it is difficult to fit some of the more complex models.

A further elaboration is possible to simultaneously estimate all the functions (such as the angles for the torso, arm, head etc.) that describe the motions. We can add an additional level of nesting to the model expressing the $l^{\text {th }}$ function, giving us the following model:

$$
y_{i j k l}=x_{i j l}^{T} \beta_{k}+z_{i l} \gamma_{i l k}+z_{i j l} \gamma_{i j k l}+\varepsilon_{i j k l}
$$

It is quite likely that different predictors might be used for the random and fixed effects depending on the function being modeled. This approach has the advantage of allowing us to model correlation between functions during motion. For example, we might suspect that the torso and arm angles show some covariation. The disadvantage is that the number of parameters that must be simultaneously estimated becomes very large and computational considerations may mean that only subsets of all the functions may be fit.

\section{EXAMPLE}

To illustrate these points, we fit a model for radial deviation which takes the form:

$$
y_{i j k}=\mu_{k}+c_{i j}^{x} \beta_{k}^{x}+c_{i j}^{y} \beta_{k}^{y}+c_{i j}^{z} \beta_{k}^{z}+c_{i j}^{x^{2}} \beta_{k}^{x^{2}}+c_{i j}^{y^{2}} \beta_{k}^{y^{2}}
$$




$$
\begin{aligned}
& +c_{i j}^{z^{2}} \beta_{k}^{z^{2}}+c_{i j}^{x} c_{i j}^{y} \beta_{k}^{x y}+c_{i j}^{x} c_{i j}^{z} \beta_{k}^{x z}+c_{i j}^{z} c_{i j}^{y} \beta_{k}^{z y} \\
& + \text { gender }_{i} \beta_{k}^{g}+\operatorname{age}_{i} \beta_{k}^{a}+\text { height }_{i} \beta_{k}^{h}+\gamma_{i}+\gamma_{i j}+\varepsilon_{i j k}
\end{aligned}
$$

where $\gamma_{i} \sim N\left(0, \sigma_{s}^{2}\right), \gamma_{i j} \sim N\left(0, \sigma_{r}^{2}\right)$ and $\varepsilon_{i j k} \sim N\left(0, \sigma_{k}^{2}\right)$. The model for the radial deviation is quadratic in the final hand coordinates $\left(c^{x}, c^{y}, c^{z}\right)$ and has linear terms in the gender, age and height (stature). There is subject variance $\sigma_{s}^{2}$, the reach variance $\sigma_{r}^{2}$ and the error variance $\sigma_{k}^{2}$ which is allowed to vary with the B-spline coefficient $k$ to allow for differing amounts of variation at different times during the reach. The first and last B-spline in the basis set are removed to ensure that the radial deviation is predicted as zero at the endpoints.

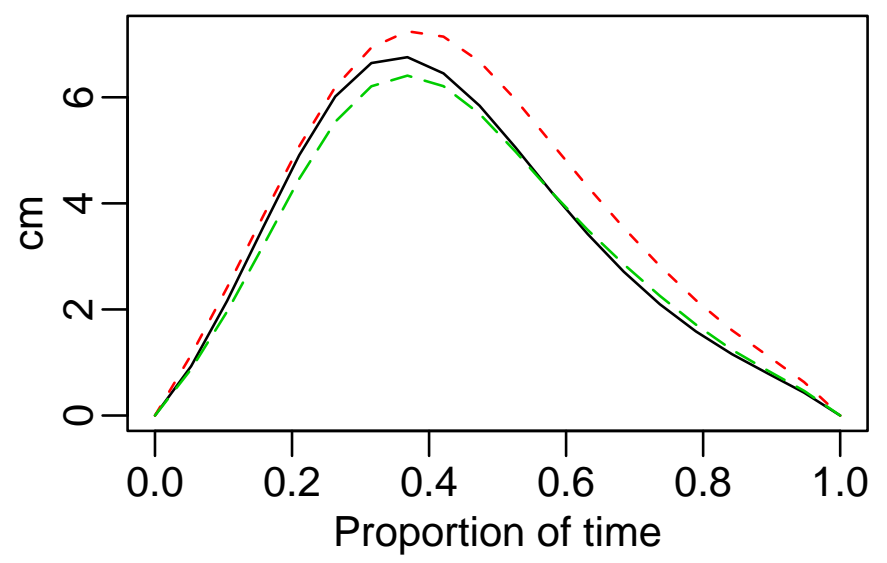

Figure 2: Predicted radial deviations for three subjects, each $167.8 \mathrm{~cm}$ tall reaching with the right hand to a location on the left of the body. The solid line is a female, 20 years old, the dashed line is a male, 20 years old and the dotted line is a female, 70 years old.

The ratio of the reach to subject estimated standard deviations is 1.6. This means that the variation in repeated reaches is somewhat greater than the variation of the average reaches of different subjects. By estimating the $\sigma_{k}^{2}$ we find that, compared to the standard deviation near the end of the reach, the standard deviation near the middle of the reach is 2.7 times greater while this same ratio drops to 0.59 near the end of the reach. Thus, as might be expected, the variability in the middle of the reach is the greatest while it is least near the end of the reach (because the hand is reaching to a specified target which constrains the motion somewhat).

All the fixed effects terms except stature are statistically significant in this model. The p-values of gender, age and stature are respectively, 0.0015, 0.0275 and 0.9246 . Note that if we ignore the grouping structure in the data and fit a model without $\gamma_{i}$ and $\gamma_{i j}$, the corresponding p-values will be incorrect since they won't allow for the correlation in the reaches. The magnitudes of these effects are illustrated in Figure 2. We see that females and older subjects show generally greater radial deviation than males and younger subjects. The differences are not constant over the reach. We have shown the predicted reaches to the same target on the left of the body as shown in Figure 1 and for a subject $167.8 \mathrm{~cm}$ tall. Since the age and gender do not have any interaction terms in the model, the relative differences in the three reaches would remain the same if we changed the target or the stature considered. 
We can visualize the effects of between and within subject variation by randomly generating reaches from the fitted random effects distribution and plotting the results. Consider a female subject, $169.8 \mathrm{~cm}$ tall and 47 years old. We can use the model above to predict the motion of such a subject on the average, but we'd also be interested in how a given subject of this type might vary in their reach and also how much subjects of this type vary. We can use a random number generator to generate simulated values of $\gamma_{i}, \gamma_{i j}$ and $\varepsilon_{i j k}$ with the variances we have estimated, to produce simulated motion. In Figure 3, we show 20 such simulated reaches.

If a person were to actually repeat a reach 20 times, there is the possibility of a learning effect - perhaps the variability might decrease over time. We don't have data of this type but we could model it by including a time term in the model, perhaps in both fixed and random effects parts. In Figure 3, we are observing 20 potential first reaches by this individual.

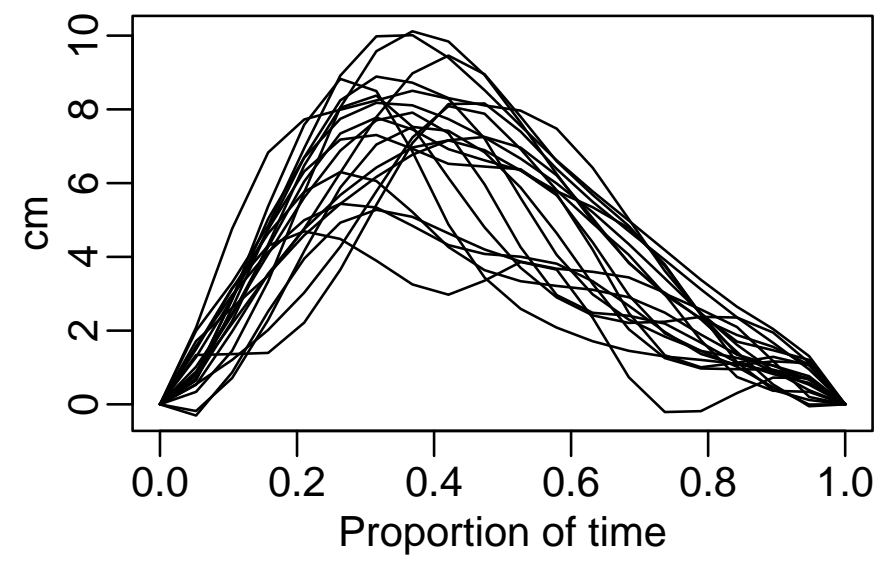

Figure 3: Within subject variability: 20 Simulated radial deviations for a given $169.8 \mathrm{~cm}$ tall, 47 year old woman

Now imagine 20 different 47 year old females each $169.8 \mathrm{~cm}$ tall. Suppose we were able to compute the average reach for each one of these women. These average reaches would not be identical because there would be personal differences beyond age, gender and stature. We can simulate this type of variation using the $\gamma_{i}$ term. We have randomly generated 20 such average reaches in Figure 4.

This between subject variation seems relatively small here. The variation might be due to factors other than age, gender and stature that we have already included in the model or it could also be due to unexplainable personal differences. In any case, there seems to be no compelling necessity to search for other factors given the small variation.

When predicting a new motion by a new subject, we would need to combine these two types of variability that we have just demonstrated. In this particular instance, the within subject variability is clearly larger but for quantities other than the radial deviation, this may not be so. 


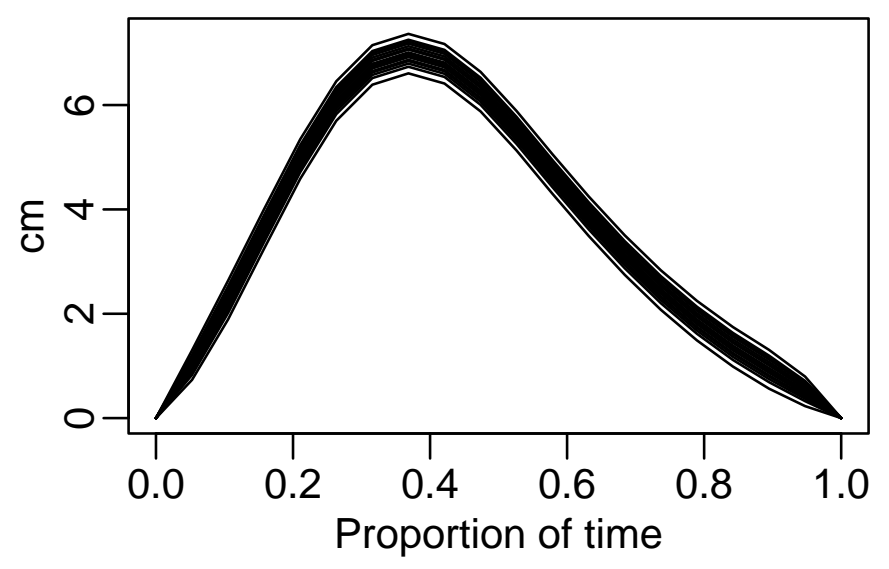

Figure 4: Between subject variability: 20 Simulated average radial deviations for 20 different $169.8 \mathrm{~cm}$ tall, 47 year old women

\section{CONCLUSION}

Point predictions of motion are useful, but a full understanding requires knowledge of the amount variability to be expected. We have demonstrated a method for modeling this variability. The possibilities of the approach are rich in number and we have only just begun to investigate them.

\section{ACKNOWLEDGMENTS}

The HuMoSim project at the University of Michigan and Don Chaffin and Bernard Martin in Rtefelénces

[1] W. G. Darling, K. J. Cole, and J.H. Abbs. Kinematic variability of grasp movements as a function of practice and movement speed. Experimental Brain Research, 73:225-235, 1988.

[2] J. Faraway. Regression analysis for a functional response. Technometrics, 39:254-261, 1997.

[3] J. Faraway. Modeling reach motions using functional regression analysis. SAE Technical Paper 2000-01-2175, 2000.

[4] S.R. Gutman and G.L. Gottlieb. Basic functions of variability of simple pre-planned movements. Biological Cybernetics, 68:63-73, 1992.

[5] R. Johnson and D. Wichern. Applied Mulivariate Statistical Analysis. Prentice Hall, New Jersey, 3rd edition, 1992.

[6] J. C. Pinheiro and D. M. Bates. Mixed-Effects Models in S and S-PLUS. Springer, New York, 2000.

[7] J. Ramsay and B. Silverman. Functional Data Analysis. Springer, New York, 1997. 
[8] R. A. Schmidt, H. Zelaznik, B. Hawkins, J. S. Franks, and J. T Quinn. Motor-output variability: A theory for the accuracy of rapid motor acts. Psychological Review, 86:415-451, 1979.

[9] Qing Shen. Linear Models for a Functional Response. PhD thesis, Department of Statistics, University of Michigan, 1998. 